\title{
Response to Letter to the Editor by Dr. Yiu Ming Wong: Pain Induced by Rheumatoid Arthritis and Acupoint Skin Resistance
}

\author{
Wolfgang Linden · Linda Turner
}

Published online: 7 June 2013

(C) Springer Science+Business Media New York 2013

We greatly appreciate the interest Dr. Yiu Ming Wong has taken in our work. Her thoughtful response points out the direction that the next set of related studies should take, and her comments can readily serve as a research agenda for such further investigations. We fully agree with her observations about unresolved issues and alternative explanations. We like to stress that we see our study as merely a first, tentative but promising, bridge between Chinese medicine concepts and Western ways to conduct validation research about them. Ideally, we like to see this mapping approach applied to all prevalent types of disease and distressing conditions and believe that a much stronger bridge can ultimately be built.

This is a response to the article doi:10.1007/s10484-013-9212-y.

W. Linden $(\varangle) \cdot$ L. Turner

UBC, Vancouver, BC, Canada

e-mail: wlinden@psych.ubc.ca 